\title{
PROTOCOLO ORTODÓNCICO EN PACIENTES CON ANTECEDENTE DE RAÍCES DE TAMAÑO DISMINUIDO EN INCISIVOS CENTRALES SUPERIORES: SERIE DE CASOS
}

\author{
${ }^{1}$ German E. Puerta Salazar, ${ }^{2}$ Santiago Herrera Guardiola \\ ${ }^{1}$ Especialista en Ortodoncia, docente U. del Valle, Cali, Colombia \\ ${ }^{2}$ Estudiante de III Año, Especialización en Ortodoncia U. del Valle, Cali, Colombia
}

Autor responsable de correspondencia: Santiago Herrera Guardiola

Correo electrónico: sahegu5@gmail.com

\begin{abstract}
RESUMEN
La resorción radicular es un efecto adverso indeseable durante el tratamiento de ortodoncia. Existen diversas causas que la producen, tales como fuerzas excesivas, morfología radicular o factores genéticos predisponentes; sin embargo, no hay suficiente evidencia científica acerca del tratamiento de ortodoncia en pacientes con el proceso de resorción como antecedente importante.

El propósito del presente artículo es darle a entender al clínico sobre la resorción radicular y su posible manejo cuando existan raíces de tamaño disminuido previo al inicio del tratamiento de ortodoncia.

Se presenta una serie de casos con raíces de tamaño disminuido de incisivos centrales superiores; en todos ellos se hace tratamiento de ortodoncia correctivo con extracciones de primeros premolares superiores e inferiores por necesidad tanto funcional de selle labial como de estética facial.

Los pacientes finalizan el tratamiento con adecuadas relaciones oclusales, funcionales y estéticas, sin detrimento del estado radicular y pulpar de los dientes afectados.

Pacientes con antecedente de tamaño radicular disminuido pueden ser sometidos a tratamiento de ortodoncia con cierre de espacios y retracción incisiva, mediante un correcto diagnóstico, eficiente biomecánica y el uso de fuerzas ligeras, sin empeorar la severidad de la longitud radicular y de la vitalidad dental. [Puerta GE, Herrera S. Protocolo ortodóncico en pacientes con antecedente de raíces de tamaño disminuido en incisivos centrales superiores: serie de casos. Ustasalud 2015;14:59-66].
\end{abstract}

Palabras clave: Resorción radicular, ortodoncia correctiva, extracción dental.

\section{ORTHODONTIC TREATMENT PROTOCOL IN PATIENTS WITH PREVIOUS SHORT ROOT LENGTH OF UPPER CENTRAL INCISORS. CASE SERIES}

\footnotetext{
ABSTRACT

Root resorption is a side effect of orthodontic treatment. There are factors involve in this phenomenon such as excessive applying forces, root anatomy or genetic predisposition. However, there are not enough literature about the orthodontic treatment on patients with severe root resorption as a previous finding.

The purpose of this report is explain to the clinician this pathology, and a possible orthodontic treatment.

We present a several cases with previous short root length of the upper central incisors. The orthodontic treatment requires the extraction of four bicuspids to improve the functional lip gap and facial aesthetics.

The patients finish the orthodontic treatment with oclusal stability, functional relationship and good aesthetics without detriment of the root condition of the upper incisors.

Patients with a history of reduced radicular size can undergo orthodontic treatment with closure of spaces and incisive retraction, through a correct diagnosis, efficient biomechanics and the use of light forces, without worsening the severity of root length and dental vitality.
}

Keywords: Root resorption, corrective orthodontics, tooth extraction. 


\section{INTRODUCCIÓN}

La resorción radicular es un efecto indeseable en cualquier tratamiento, ya que afecta su viabilidad, y su pronóstico disminuye a largo plazo. Según lo reportado en la literatura los pacientes que se encuentran bajo tratamiento ortodóncico tienen mayor riesgo de acortamiento apical severo ${ }^{1-15}$. Se deben realizar controles una vez finalizado el tratamiento de ortodoncia para determinar la evolución de los dientes afectados. Puerta y Herrera reportan el manejo ortodóncico de un paciente que presentó reabsorción radicular previa, con buenos resultados oclusales, funcionales y estéticos ${ }^{16}$. Adicionalmente, se reporta un caso tratado ortodóncicamente que presentó reabsorción radicular durante el tratamiento y una vez finalizado el tratamiento se hizo el respectivo seguimiento de 25 años, encontrándose radiográficamente que no aumentó la reabsorción radicular ${ }^{15}$.

\section{DIAGNÓSTICO}

Se puede diagnosticar la resorción radicular mediante técnicas radiológicas, a partir de una toma inicial y subsecuentes registros durante el movimiento dental, siempre y cuando se realicen bajo una técnica estandarizada y un equipo radiológico con las mismas características. En dientes de riesgo aumentado, como ápices romos o en forma de pipeta, se recomienda el estudio radiográfico cada tres meses ${ }^{15,17,18}$. Las radiografias periapicales están indicadas como mejor método diagnóstico comparado con la radiografía panorámica, debido a la menor distorsión de imagen que presentan las primeras; sin embargo, Dudic y colaboradores ${ }^{18}$ sugirieron que la radiografia periapical digitalizada presenta menor sensibilidad para diagnosticar la reabsorción comparada con la micro-tomografia computarizada. La tomografía computarizada de haz cónico es un excelente método de diagnóstico, debido a que ofrece una visión en 3D y minimiza las dosis de radiación comparado con un juego periapical completo. La principal limitación de este método podría ser la posible dosis de radiación que recibe el paciente y su costo económico, no obstante, estos factores han sido minimizados con la baja emisión de radiación de los nuevos tomógrafos y la disminución de costos radiográficos.

\section{Clasificación}

Andreasen clasificó la reabsorción radicular externa en tres tipos de acuerdo con su etiología y patogenia:

- Reabsorción de superficie, causada por la agresión al ligamento periodontal.

- Reabsorción inflamatoria, consecuencia de la combinación entre la agresión al ligamento perio- dontal y la superficie radicular con la presencia de bacterias en el canal radicular.

- Reabsorción por sustitución, debida a la fusión entre el hueso adyacente y la superficie radicular ${ }^{19}$.

Actualmente la clasificación más utilizada es la de Malmgren y colaboradores de 1982, pero modificada por Janson y colaboradores en el 2000, dado que usan índices cuantitativos para catalogar los diferentes estados en: ausente, leve, moderada, acentuada y severa ${ }^{20,21}$.

\section{Factores etiológicos}

De acuerdo con la línea de investigación del Dr. Darendeliler ${ }^{2,3,5-10,12,14,22}$, quien presenta un protocolo establecido y una apropiada metodología para determinar si se presenta la reabsorción radicular en diferentes situaciones ortodóncicas, se concluye que los dientes que son tratados con fuerzas pesadas tienden a presentar una mayor cantidad de lagunas o cráteres de resorción. Con una relación de presencia de reabsorción radicular diez veces mayor con fuerzas pesadas y cuatro veces mayor con fuerzas ligeras en comparación con un grupo control. La región apical junto a la zona de presión donde se dirige el movimiento son las zonas donde se presentan los procesos de resorción. De igual forma, comparando fuerzas intermitentes y contínuas, se concluye que en las fuerzas intermitentes presentaron menor cantidad de resorción radicular de forma significativa ${ }^{2,3}$.

Otros factores de riesgo reportados de reabsorción radicular en incisivos superiores están relacionados con la historia de traumas dentoalveolares, historia de tratamientos de ortodoncia de larga duración, biotipo óseo delgado, proporción corona raíz disminuida y raíces con forma triangular, en pipeta 0 dilacerada ${ }^{23,24}$.

Finalmente, los factores genéticos predisponentes son dificiles de identificar y solo en aquellos pacientes que presentan historia familiar de reabsorción con ortodoncia, o en casos de agenesia de 4 o más dientes podría afirmarse que existe mayor riesgo de reabsorción radicular ${ }^{25}$.

El propósito del presente artículo es presentar un posible manejo ortodóncico cuando se tiene antecedentes de raíces de tamaño disminuido previo al tratamiento de ortodoncia en casos de extracciones dentales.

\section{PRESENTACIÓN DE CASOS}

Todos los casos fueron tratados con sistemas de autoligado interactivo con prescripción Roth y arcos de ortodoncia con características fisicas térmicas y de súper elasticidad, los cuales proporcionan fuerzas leves. 


\section{Protocolo de manejo ortodóncico}

El protocolo de tratamiento el cual proponemos es, a partir de un correcto proceso diagnóstico oclusal $\mathrm{y}$ funcional, un manejo de ortodoncia con exodoncias de primeros premolares para lograr una adecuada relación funcional muscular de la zona neutral ${ }^{26} \mathrm{y}$ adecuada estética dental. Se inician los casos con las exodoncias previamente realizadas. Se procede con una adecuada fase de nivelación y alineación de los arcos, pero no se involucran al arco los dientes con reabsorción radicular previa. Una vez se encuentre en arcos de acero de trabajo se realiza mecánica intra-arco activa para el cierre de los espacios, además de mecánica elástica intermaxilar con vector de clase II, cabe anotar que si los caninos superiores se encuentran afectados por el proceso de reabsorción no se deben situar los elásticos en estos, se deben situar en un hook soldado entre canino e incisivo lateral. Ya logrado el cierre de los espacios de extracción se involucran al arco los dientes afectados previamente por la reabsorción, en este caso arcos de alineación y nivelación térmicos súper elásticos, y lograr una adecuada estética dental. Se finalizan los casos con parámetros estéticos adecuados y parámetros funcionales, tanto oclusales como musculares, que mejoren el pronóstico del paciente.

\section{Secuencia biomecánica}

1. Alineación: arcos térmicos 0.13 (CuNiTi, Ormco, USA) o 0,14 (Sentalloy, GAC, USA) según la cantidd de apiñamiento pre-existente. Una vez corregido el apiñamiento incial pasar a arcos 0,18 (Sentalloy, GAC, USA) o 0,16 x 0,16 (Bioforce, GAC, USA). Se finaliza la fase de alineación y nivelación mediante arcos 0,16 x 0,22 (Bioforce, GAC, USA) o $0,17 \times 0,25$ (Neosentalloy, GAC, USA).

2. Fase de trabajo: $\operatorname{arcos} 0,17 \times 0,25$ de acero coordinados transversalmente. Se esperan cuatro semanas para iniciar la mecánica de cierre de espacios mediante la activación de fuerzas ligeras con sistemas elásticos tipo tie-back o con resortes de NiTi. Se recomienda el uso de anclaje dentoalveolar para consolidar el segmento anterior durante el cierre de los espacios.

3. Fase de re-nivelación y finalización: una vez logrado el cierre total de los espacios de extracción se cementan los brackets de los dientes afectados, uso de arcos 0,18 (Sentalloy, GAC, USA) térmicos durante cuatro semanas. Arcos $0,20 \times 0,20$ o $0,16 \times 0,22$ (Bioforce, GAC, USA) para detallado y asentamiento final.

\section{Caso \# 1}

Paciente de sexo femenino de 28 años de edad quien no refiere antecedentes médicos y odontológicos de importancia. Con diagnóstico de clase II esquelética, biotipo dolicofacial, maloclusión dental de clase II molar derecha con subdivisión, biprotrusión de incisivos, mordida abierta. Al análisis funcional se reporta hábito de interposición lingual e incompetencia labial, redundancia de tejidos blandos periorales. Al examen tomográfico se observa una disminución de la longitud radicular de dientes incisivos superiores e inferiores previo al tratamiento de ortodoncia. Se hace uso de aparatología fija de autoligado interactivo estético prescripción Roth (In-Ovation C, GAC, USA). Una vez finalizado el tratamiento no se observan cambios significativos en cuanto a la longitud radicular. El tratamiento tuvo una duración de 26 meses (Figuras 1, 2 y 3).

\section{Caso \# 2}

Paciente de sexo femenino, 11 años de edad con diagnóstico de clase II esquelética, maloclusión dental clase I con apiñamiento moderado, mordida abierta y biprotrusión dentoalveolar. Funcionalmente presenta incompetencia labial, y un desbalance muscular de la zona neutral (Figura 4). Se hace uso de aparatología fija de autoligado interactivo estético prescripción Roth (In-Ovation C, GAC, USA). Se finaliza el proceso de ortodoncia en catorce meses, aunque no finaliza el tratamiento debido a cambio de domicilio, faltándole un $10 \%$ para culminar el tratamiento por completo. Se obtienen guías funcionales, relación muscular y adecuada estética a pesar de no finalizar su tratamiento por completo (Figura 5).

\section{Caso \# 3}

Se presenta un caso de sexo femenino de 16 años de edad. Clase II esquelética, biotipo dolicofacial y tendencia a mordida abierta. Maloclusión dental clase I con apiñamiento severo en arco superior y moderado inferior, mordida abierta de 13 a 23, biprotrusión dentoalveolar. De nuevo la función muscular se ve comprometida y no hay una adecuada relación de los músculos orbiculares orales (Figura 6).

Se hace uso de aparatología fija de autoligado interactivo estético prescripción Roth (In-Ovation C, GAC, USA). Se realiza el manejo con exodoncias y de acuerdo con el protocolo presentado, se programan exodoncias de primeros premolares previo al inicio de los arcos de alineación y nivelación. Se hacen controles radiográficos trimestrales durante el cierre de espacios para valorar el grado de reabsorción radicular. No se involucran los incisivos afectados hasta completar el cierre total del espacio de extracción (Figura 7). 


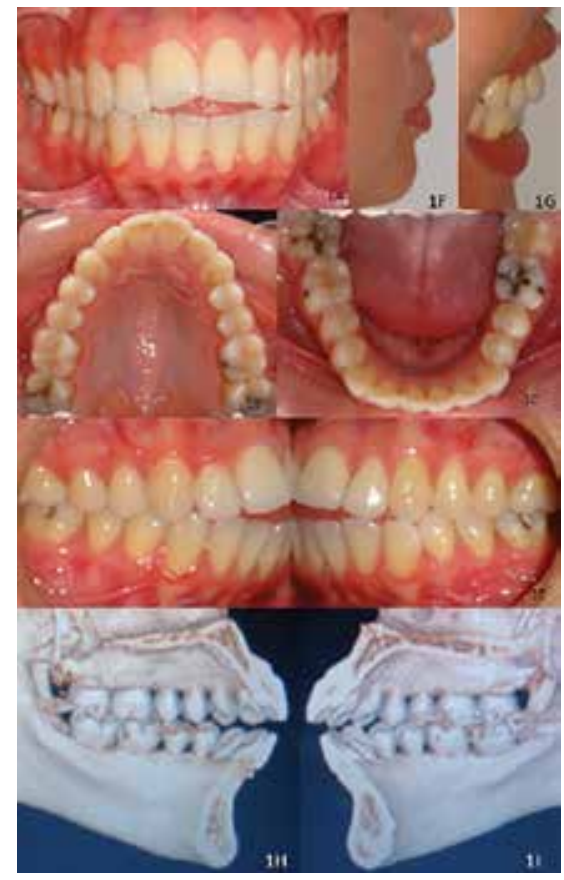

Figura 1. Fotografias paciente 1 previas al tratamiento. Panorámica intraoral (A), Oclusal superior (B), Oclusal inferior (C), Lateral derecha (D), Lateral izquierda (E), Extraoral perfil de emergencia reposo (F) y sonrisa (G), nótese desbalance de la zona neutral. Tomografia pretratamiento diente $11(\mathrm{H})$ y diente 21 (I); Reabsorción radicular severa

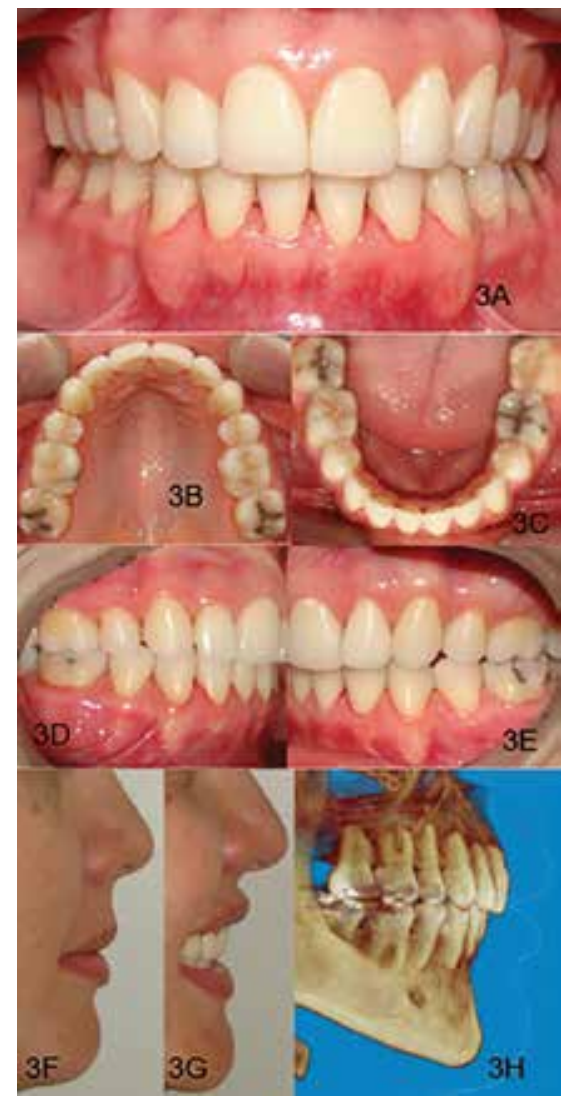

Figura 3. Fotografia finalización paciente 1. Panorámica intraoral (A). Oclusal superior (B) y Oclusal inferior (C). Lateral derecha (D), e izquierda $(E)$. Fotografia extraoral perfil de emergencia en reposo (F) y sonrisa (G); nótese la armonía de los músculos periorales y la adecuada disposición labial en reposo. Tomografia de finalización (H), no se aprecian cambios en la distancia radicular.

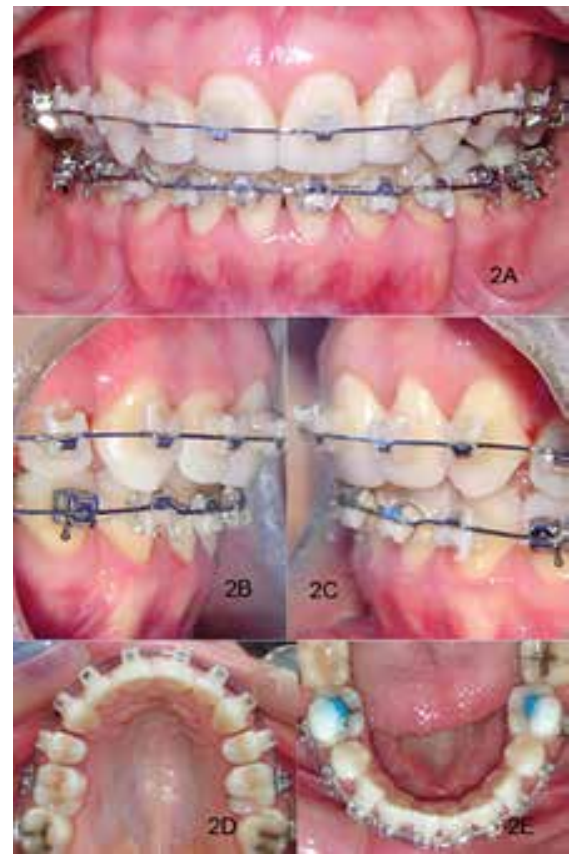

Figura 2. Paciente 1 Fase de re-nivelación y alineación con arco térmico $0.20 \times 0.20$ superior. Fotografia panorámica intraoral (A). Fotografia lateral derecha (B) e izquierda (C). Oclusal superior (D) e inferior (E).

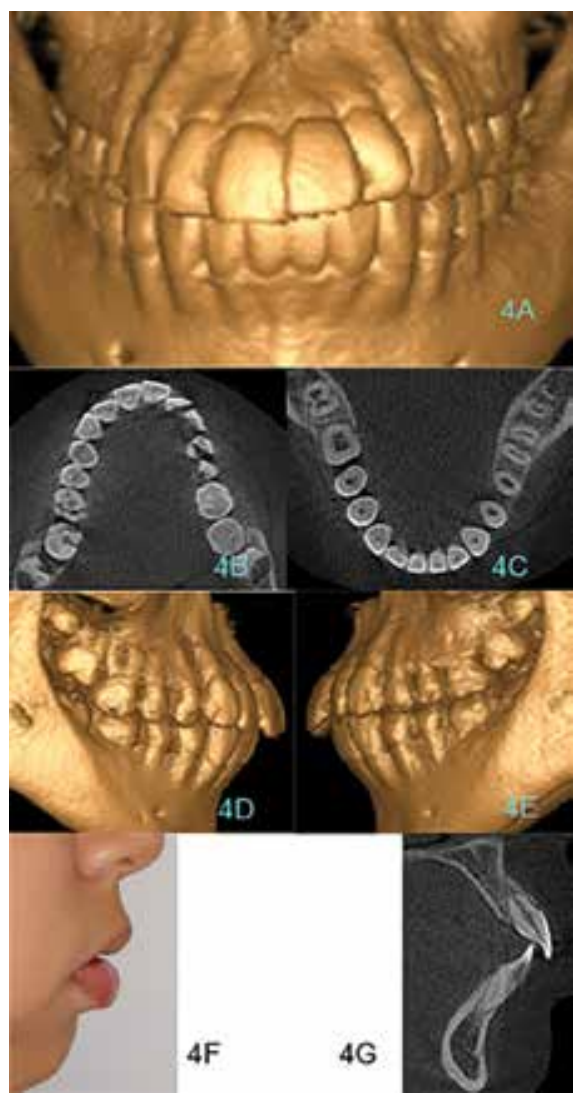

Figura 4. Cortes tomográficos previos al tratamiento paciente 2. Panorámica (A), Oclusal superior (B), Oclusal inferior (C), Lateral derecha (D), Lateral izquierda (E), Fotografia extraoral perfil de emergencia reposo (F); se observa desbalance de la zona neutral; incompetencia labial y eversión del labio inferior. Corte sagital de dientes 11 y $41(\mathrm{H})$; Reabsorción radicular severa. 


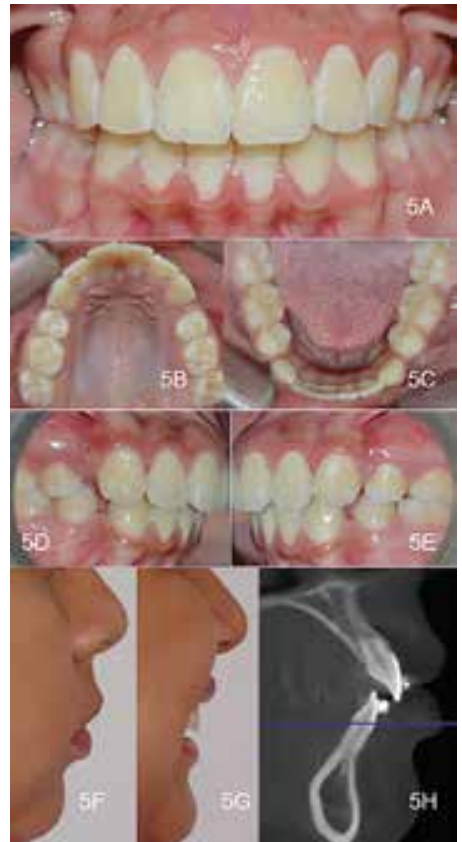

Figura 5. Paciente 2 fotografía panorámica intraoral postratamiento (A). Fotografía final oclusal intraoral superior (B), e inferior (C). Fotografia lateral derecha (D) e izquierda (E) final. Fotografia extraoral final del perfil de emergencia en reposo $(F)$ y sonrisa $(G)$; se observa una mejor disposición labial en reposo y una adecuada proyección de los incisivos superiores. Tomografia final del tratamiento $(\mathrm{H})$, no hay cambios de la longitud radicular.

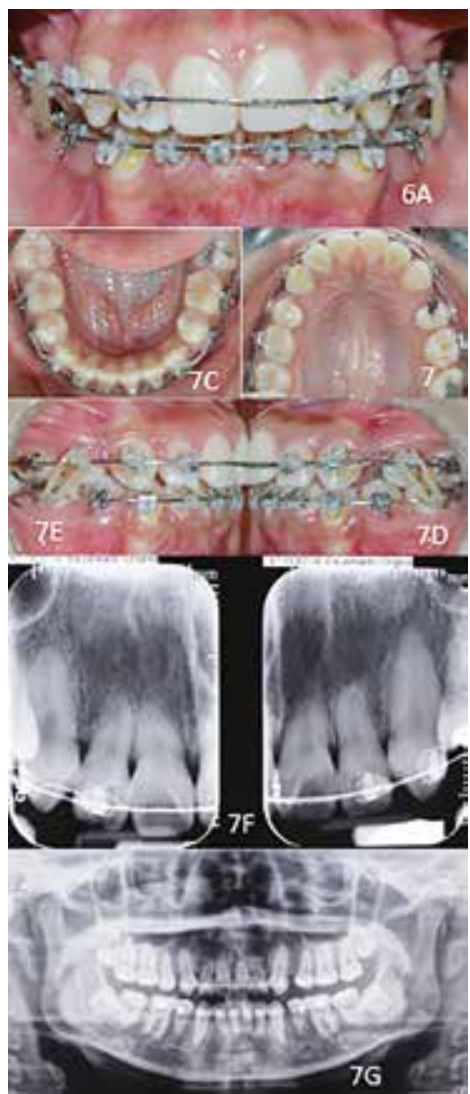

Figura 7. Fotografias intermedias al tratamiento de paciente 3. Panorámica intraoral (A), Oclusal superior (B), Oclusal inferior (C), Lateral derecha (D), Lateral izquierda (E). Radiografía periapical intermedia al tratamiento de dientes 11 y 21 (F) Panorámica intermedia (G); se mantiene la reabsorción radicular severa.

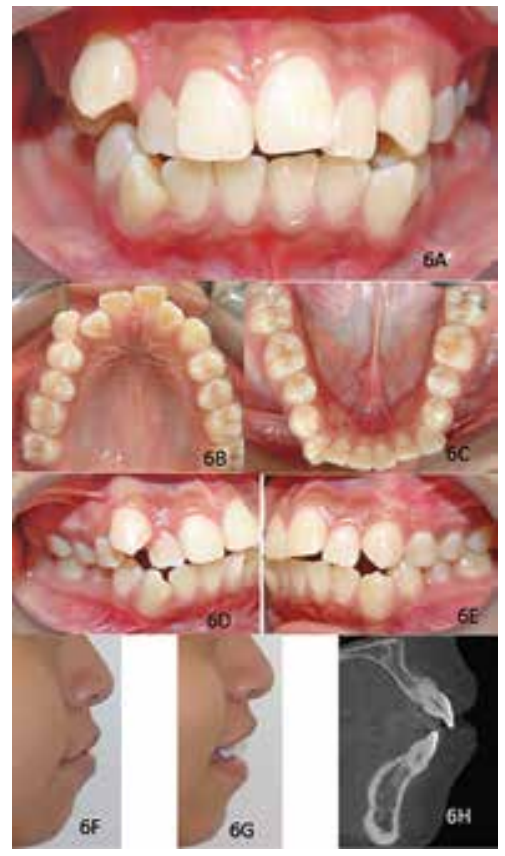

Figura 6. Fotografias previas al tratamiento paciente 3. Panorámica intraoral (A), Oclusal superior (B), Oclusal inferior (C), Lateral derecha (D), Lateral izquierda (E), Extraoral perfil de emergencia reposo $(F)$ y sonrisa (G), se observa desbalance de la zona neutral; incompetencia labial y eversión del labio inferior. Tomografía pretratamiento diente 11 y 41 $(\mathrm{H})$; Reabsorción radicular severa.

\section{Caso \# 4}

Paciente de 15 años de edad que ingresa a las clínicas de ortodoncia de la Universidad del Valle sin antecedentes médicos y odontológicos de relevancia; sin embargo, la madre reporta como antecedente trauma dento-alveolar de incisivos superiores a los 8 años de edad. Al examen clínico se encontraron los incisivos asintomáticos y con movilidad fisiológica. Al análisis facial presenta línea de labio superior invertida, biproquelia y ausencia del selle labial en reposo. Maloclusión dental clase I molar y clase II canina con proinclinación de incisivos. Al examen radiológico panorámico los dientes 11 y 21 presentan raíces cortas, ápices achatados y cresta ósea a nivel de tercio medio radicular (Figuras $8 \mathrm{y}$ 9). Se hace uso de aparatología fija de autoligado interactivo estético prescripción Roth (In-Ovation R, GAC, USA). El tratamiento activo completo tuvo una duración de 18 meses (Figura 10).

\section{DISCUSIÓN}

Varios autores reportan que la resorción radicular es un efecto deletéreo durante el tratamiento de ortodoncia. Según lo manifestado por Lupi y colaboradores $^{27}$ en su estudio con una muestra poblacional étnicamente diversa, se presentó la resorción radicular externa de incisivos en $15 \%$ de la población antes del inicio del tratamiento de ortodoncia y del 
$73 \%$ después de 12 meses. Antes del inicio del tratamiento, el $2 \%$ de incisivos presentaron reabsorción moderada - severa, y después del tratamiento se presentó la reabsorción en el $24,5 \%$ de los incisivos. Lavender y Malgrem ${ }^{28}$ reportaron una incidencia del $34 \%$, luego de seis meses de haber iniciado el tratamiento, al finalizar la frecuencia aumentó al 56\%. Adicionalmente, Sehr y colaboradores informaron que la incidencia de casos después del tratamiento ortodóncico es tan solo del $0,5 \%$ pero este estudio presentó una deficiencia metodológica, debido al método de diagnóstico con radiografía panorámica que tiene un gran porcentaje de distorsión ${ }^{29}$. Gonzáles y colaboradores publicaron en 2012 un estudio de cohortes con seguimiento a un año, en el que encuentran que existe una incidencia de reabsorción radicular del $68,2 \%$ entre los 9 y 11 meses de haber iniciado el tratamiento de ortodoncia. La técnica de canto tuvo una probabilidad 3,3 veces de presentar reabsorción radicular al compararlo con técnicas preajustadas ${ }^{30}$. Se reporta que movimientos de intrusión y torque presentan gran magnitud de reabsorción radicular ${ }^{10,12,31}$, sin embargo, el Dr. Darendeliler publicó que es posible encontrar lagunas de resorción radicular, en terceros molares no erupcionados, mediante el método de micro tomografia computarizada, pero esas lagunas son menores que en dientes tratados ortodóncicamente ${ }^{22}$.

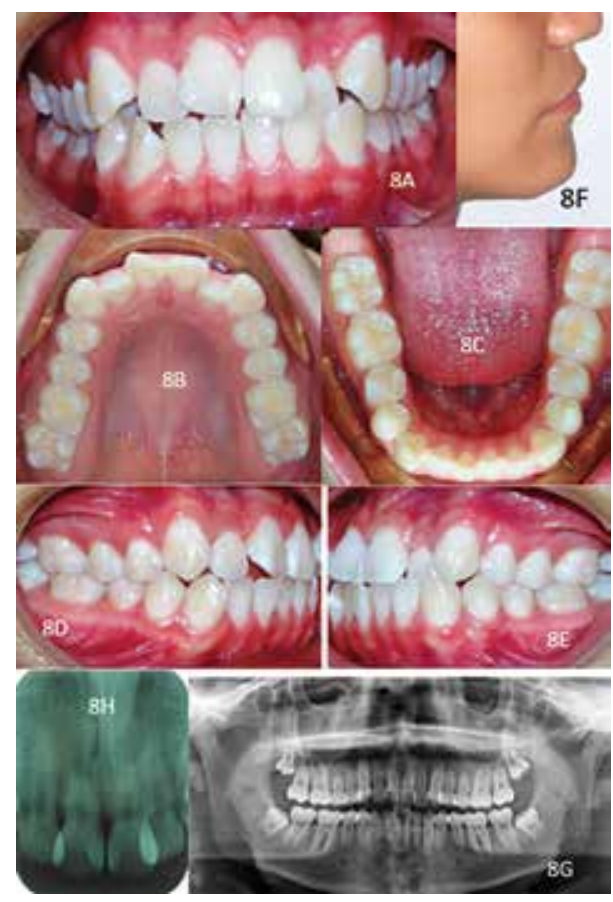

Figura 8. Paciente 4, fotografia panorámica intraoral previo al tratamiento (A). Fotografía inicial oclusal intraoral superior (B), e inferior (C). Fotografia lateral derecha (D) e izquierda (E) inicial. Fotografia extraoral inicial del perfil de emergencia en reposo (F); se observa una disposición de hiperfunción de músculos periorales en reposo. Radiografia panorámica inicial del tratamiento (G), Radiografia periapical inicial de zona incisivos $(\mathrm{H})$ disminución de la longitud radicular.

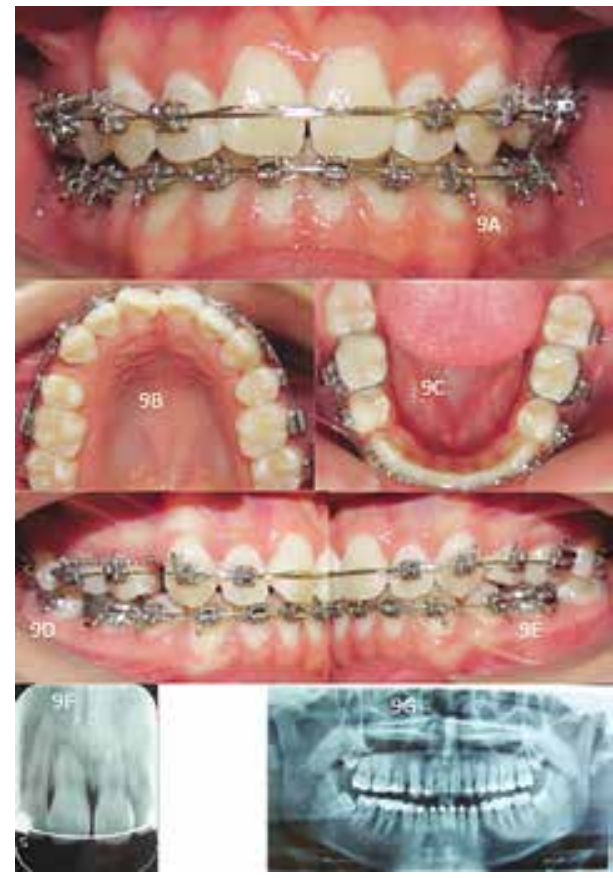

Figura 9. Registros intermedios durante la fase de trabajo de paciente 4. Fotografia intraoral panorámica (A). Oclusal superior (B) e inferior (C). Lateral derecha (D) e izquierda (E). Radiografia periapical intermedia del tratamiento $(\mathrm{F})$, y Panorámica intermedia al tratamiento $(G)$; no hay cambios en comparación con la radiografia inicial.

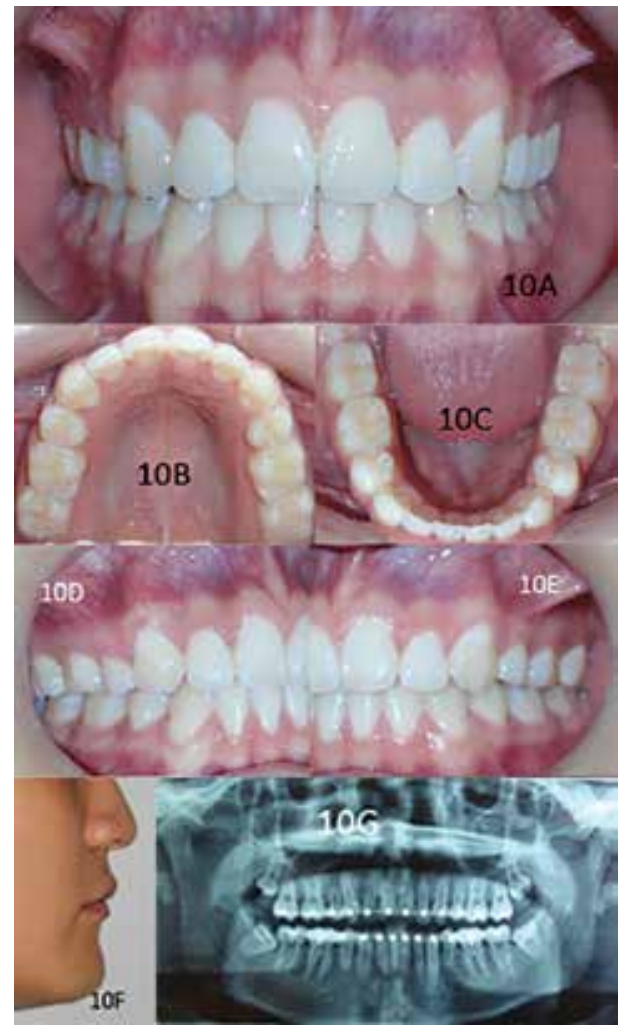

Figura 10. Paciente 4, fotografía panorámica intraoral final al tratamiento (A). Fotografia final oclusal intraoral superior (B), e inferior (C). Fotografia lateral derecha (D) e izquierda (E) final. Fotografia extraoral final del perfil de emergencia en reposo $(\mathrm{F})$; se observa una mejor disposición de músculos periorales en reposo. Radiografía panorámica final del tratamiento $(G)$; no hay cambios en la disminución de la longitud radicular. 
Un correcto diagnóstico del tamaño radicular debe realizarse mediante tomografia computarizada 0 en su defecto radiografias periapicales que tienen menor distorsión que la radiografia panorámica y los controles deben hacerse una vez iniciado el tratamiento cada tres a cuatro meses ${ }^{13,17}$.

La magnitud de la fuerza empleada es un factor muy importante en la aparición de lagunas de resorción radicular. Según las investigaciones de Paetyangkul desde 0,25 newtons pueden encontrarse lagunas de resorción y con fuerzas mayores $(2,25 \mathrm{~N})$ se encontró mayor cantidad y magnitud de cráteres resortivos de manera estadísticamente significativa ${ }^{9}$.

Se ha reportado que mediante la estimulación con ultrasonido se puede reducir la posibilidad de reabsorción radicular dado por fuerzas ortodónci$\mathrm{Cas}^{32}$. Lo anterior es posible dado que se suprime la cementoclastogénesis alterando la relación de OPG/ RANKL durante el proceso de ortodoncia sin alterar los movimientos dentales, sin embargo, se requieren más estudios que avalen el uso de ultrasonido. Una variable ampliamente discutida es el tiempo de duración del tratamiento, donde a mayor tiempo de exposición de fuerzas puede ocurrir una mayor magnitud de reabsorción radicular ${ }^{3,8,9}$. Las fuerzas ortodóncicas de los incisivos centrales afectados, de la presente serie de casos, fueron en lo menor posible en tiempo y en magnitud. De igual forma, se realizaron controles con radiografias periapicales y tomográfias de Cone Beam.

\section{CONCLUSIONES}

Pacientes con antecedente de tamaño radicular disminuido pueden ser sometidos a tratamiento de ortodoncia con cierre de espacios y retracción incisiva, mediante un correcto diagnóstico, eficiente biomecánica y el uso de fuerzas ligeras, sin empeorar la severidad de la longitud radicular y de la vitalidad dental.

Lograr una adecuada relación de la zona neutral proporciona un mejor pronóstico funcional a los pacientes sometidos a tratamiento de ortodoncia.

Es imperativo realizar controles radiológicos cada tres a cuatro meses durante el tratamiento activo de ortodoncia en los dientes afectados, con el objetivo de hacer el adecuado seguimiento del antecedente.

\section{REFERENCIAS}

1. Belinda W, Katherine WLV, Henry WF, Shiva S, Eloise EK. Root resorption associated with orthodontic tooth movement: A systematic review. Am J Orthod Dentofacial Orthop. 2010;137:462-76.

2. Ballard DJ, Jones AS, Petocz P, Darendeliler MA. Physical properties of root cementum: Part 11. Continuous vs in- termittent controlled orthodontic forces on root resorption. A microcomputed-tomography study. Am J Orthod Dentofacial Orthop. 2009;136(1):e1-8.

3. Aras B, Cheng LL, Turk T, Elekdag-Turk S, Jones AS, Darendeliler MA. Physical properties of root cementum: part 23. Effects of 2 or 3 weekly reactivated continuous or intermittent orthodontic forces on root resorption and tooth movement: a microcomputed tomography study. Am J Orthod Dentofacial Orthop. 2012;141(2):29-37.

4. Srivicharnkul P, Kharbanda OP, Swain MV, Pectocz P, Darendeliler MA. Physiscal properties of root cementum: Part 3. Hardness and elastic modulus after application of light and heavy forces. Am J Orthod Dentofacial Orthop. 2005;127(2):168-76.

5. Chan E, Darendeliler MA. Physical properties of root cementum: Part 5. Volumetric analysis of root resorption craters after application of light and heavy orthodontic forces. Am J Orthod Dentofacial Orthop. 2005;127(2):18695.

6. Harris DA, Jones AS, Darendeliler MA. Physical properties of root cementum: Part 8. Volumetric analysiis of root resorption craters after application of controlled intrusive light and heavy orthodontic forces: a microcomputed tomography scan study. Am J Orthod Dentofacial Orthop. 2006;130(5):639-47.

7. Barbagallo LJ, Jones AS, Petocz P, Darendeliler MA. Physical properties of root cementum: Part 10. Comparison of the effects of invisible removable thermoplastic appliances with light and heavy orthodontic forces on premolar cementum. A microcomputed tomography study. Am J Orthod Dentofacial Orthop. 2008;133(2):218-27.

8. Chen LL, Turk T, Elekdag-Turk S, Jones AS, Petocz P, Darandeliler MA. Physical properties of root cementum: Part 13. Repair of root resorption 4 and 8 weeks after the application of continuous light and heavy forces for 4 weeks: a microcomputed tomography study. Am J Orthod Dentofacial Orthop. 2009;136(3):e1-10.

9. Paetyangkul A, Turk T, Elekdag-Turk S, Jones AS, Petocz P, Cheng LL et al. Physical properties of root cementum: Part 16. Comparisons of root resorption and resorption craters after the application of light and heavy continuous and controlled orthodontic forces for 4, 8, and 12 weeks. Am J Orthod Dentofacial Orthop. 2011;139(3):27984.

10. Bartley N, Turk T, Colak C, Elekdag-Turk S, Jones AS, Petocz $\mathrm{P}$ et al. Physical properties of root cementum: Part 17. Root resorption after the application of 2.5 and 15 of buccal root torque for 4 weeks: a microcoputed tomography study. Am J Orthod Dentofacial Orthop. $2011 \mid ; 139(4):$ e353-60.

11. Wu AT, Turk T, Colak C, Elekdag-Turk S, Jones AS, Petocz P, et al. Physical properties of root cementum: Part 18. The extent of root resorption after the application of light and heavy controlled rotational orthodontic forces for 4 weeks: a microcomputed tomography study. Am J Orthod Dentofacial Orthop. 2011;139(5):495-503.

12. Montenegro VC, Jones A, Petocz P, Gonzales C, Darendeliler MA. Physical properties of root cementum: Part 22. Root resoption after the application of light and heavy extrusive orthodontic forces: a microcomputed tomography study. Am J Orthod Dentofacial Orthop. 2012;141(1):e1-9.

13. Vaquero P, Perea B, Labajo E, Santiago A, García F. Reabsorción radicular durante el tratamiento ortodóncico: causas y recomendaciones de actuación. Cient Dent. 2011;8(1):61-70. 
14. Malek S, Darendeliler MA, Swain MV. Physical properties of root cementum: Part I. A new method for 3-dimensional evaluation. Am J Orthod Dentofacial Orthop. 2001;120(2):198-208.

15. Silva-Marques L, Teixeira-Chaves KC, Rey AC, Pereira LJ, de Oliveira-Ruelas AC. Severe root resorption and orthodontic treatment: clinical implications after 25 years of follow up. Am J Orthod Dentofacial Ortop. 2011;139:s166-9.

16. Puerta G, Herrera-Guardiola S. Tratamiento de ortodoncia con extracciones en paciente con antecedentes de reabsorción radicular severa. Reporte de caso. Revista Científica de la Sociedad Colombiana de Ortodoncia. 2014;1(1):61-70.

17. Yu JH, Shu KW, Tsai MT, Hsu JT. A cone-beam computed tomography study of orthodontic apical root resorption. J Dent Sci. 2013;8(1):74-79.

18. Dudic A, Giannopoulou C, Leuzinger M, Kiliaridis S. Detection of apical root resorption after orthodontic treatment by using panoramic radiography and cone-beam computed tomography of super high resolution. Am J Orthod Dentofacial Orthop. 2009; 135:434-7.

19. Andreasen JO, Andreasen FM. Textbook and color atlas of traumatic injuries to the teeth. 3rd ed. Copenhagen: Munksgaard; 1994.

20. Levander E, Malmgren O. Evaluation of the risk of root resorption during orthodontic treatment: a study of upper incisiors. Eur J Orthod. 1988;10:30-8.

21. Janson GRP, de Luca Canto G, Rodriguez D, Castanha JF, de Freitas MR. A radiographic comparison of apical root resorption after orthodontic treatment with 3 different fixed appliance techniques. Am J Orthod Dentofacial Orthop. 2000;118(3):262-73.

22. Deane S, Jones AS, Petocz P, Darendeliler MA. Physical properties of root cementum: Part 12. The incidence of physiologicroot resorption on unerupted third molars and its comparison with orthodontically treated premolares. A microcomputed tomography study. Am J Orthod Dentofacial Orthop. 2009;136:e148-9.

23. Sameshima GT. Predicting and preventing root resoprtion: Part I. Diagnostics factors. Am J Orthod Dentofacial Orthop. 2001;119:505-10.
24. Picanco GV, de Freitas KM, Cançado RH, Valarelli FP, Feijao CP. Predisposing factors to severe external root resorption associated to orthodontic treatment. Dental Press J Orthod. 2013;18(1):110-20.

25. Blake M, Woodside DG, Pharoah MJ. A radiographic comparison of apical root resorption after orthodontic treatment whit the edgewise and Speed appliances. Am J Orthod Dentofacial Orthop. 1995;108(1):76-84.

26. Dawson P. Functional Oclussion from TMJ to smile desing. 1st ed. St. Louis: Mosby Elsevier; 2006.

27. Lupi JE, Handelman CS, Sadowsky c. Prevalence and severity of apical root resorption and alveolar bone loss in orthodontically treated adults. Am J Orthod Dentofacial Orthop. 1996;109(1):28-37.

28. Levander E, Malmgren A. Apical root resorption during orthodontic treatment of patients with multiple aplasia: a study of maxillary incisors. European Journal of Orthodontics. 1998;20:427-34.

29. Sehr K, Bock NC, Serbesis C, Honemann M, Ruf S. Severe external apical root resorption-local cause or genetic predisposition? J Orofac Orthop. 2011;72:321-31.

30. González F, Robles V, Rivero L, Palis M, Pulido J. Reabsorción radicular inflamatoria en sujetos con tratamiento ortodóntico. Cartagena (Colombia). Rev Salud Uninorte. 2012;28(3):382-90.

31. Costopoulos G, Nanda R. An evaluation of root resorption incident to orthodontic intrusion. Am J Orthod Dentofacial Orthop. 1996;109:543-8.

32. Inubushi T, Tanaka E, Rego Eb, Ohtani J, Kawazoe A, Tanne $\mathrm{K}$ et al. Ultrasound stimulation attenuates resorption of tooth root induced by experimental force application. Bone. 2013;53(2):497-506. $x$

\section{Correo electrónico de los autores}

German Eduardo Puerta Salazar: doctordoor@gmail.com Santiago Herrera Guardiola: sahegu5@gmail.com 